\title{
Optical spectroscopy of isolated planetary mass objects in the $\sigma$ Orionis cluster ${ }^{\star}$
}

\author{
D. Barrado y Navascués ${ }^{1,2}$, M. R. Zapatero Osorio ${ }^{3}$, V. J. S. Béjar ${ }^{4}$, R. Rebolo ${ }^{4,5}$, E. L. Martín ${ }^{6}$, \\ R. Mundt ${ }^{2}$, and C. A. L. Bailer-Jones ${ }^{2}$ \\ 1 Departamento Física Teórica, C-XI-506, Universidad Autónoma de Madrid, 28049 Madrid, Spain \\ 2 Max-Planck-Institut für Astronomie, Königstuhl 17, 69117 Heidelberg, Germany \\ 3 Division of Geological \& Planetary Sciences, California Institute of Technology, MS 150-21, Pasadena, \\ CA 91125, USA \\ 4 Instituto de Astrofísica de Canarias, 38205 La Laguna, Tenerife, Spain \\ 5 Consejo Superior de Investigaciones Científicas, CSIC, Spain \\ ${ }^{6}$ Institute of Astronomy, University of Hawaii at Manoa, 2680 Woodlawn Drive, Honolulu, HI 96822, USA
}

Received 29 May 2001 / Accepted 15 August 2001

\begin{abstract}
We have obtained low resolution optical spectra of 15 isolated planetary mass objects (IPMOs) in the $\sigma$ Orionis cluster, and derived spectral types by comparison with nearby $\mathrm{M}$ and $\mathrm{L}$ dwarfs. The spectral types are in the range late $\mathrm{M}$ - mid $\mathrm{L}$, in agreement with our expectations based on colors and magnitudes for bona fide members. Therefore, most of these objects have masses below the deuterium burning limit. About $2 / 3$ show $\mathrm{H} \alpha$ in emission at our spectral resolution. From our spectroscopic and photometric data, we infer that three IPMOs in this sample may be binaries with components of similar masses. These results confirm that the substellar mass function of the $\sigma$ Orionis cluster, in the form $\mathrm{d} N / \mathrm{d} M$, keeps rising in the planetary domain.
\end{abstract}

Key words. giant planet formation - open clusters and associations: individual: $\sigma$ Orionis - stars: brown dwarfs

\section{Introduction}

Very recently, we have discovered 18 very faint, red objects in the $\sigma$ Orionis open cluster ( $\sim 5 \mathrm{Myr}, 352 \mathrm{pc})$, using optical and infrared photometry (Zapatero Osorio et al. 2000). If they are indeed members of the association, their masses would be below $18 M_{\text {Jupiter }}\left(1047 M_{\text {Jupiter }}=1 M_{\odot}\right)$. Other low mass objects have been found in the young cluster IC 348 (Najita et al. 2000) and in the Trapezium (Lucas \& Roche 2000; Lucas et al. 2001). They are quite intriguing, since those with masses below $13 M_{\text {Jupiter }}$ would be unable to sustain any nuclear reaction at any time (in particular deuterium fusion). Therefore, they would have masses in the planetary domain and some authors have dubbed them free-floating planets, non-fusors, or isolated planetary mass objects (IPMOs). These names rely on the mass of the objects, which can be estimated from observations and comparisons with models, whereas their origin or formation mechanism cannot be known for sure. Note that these mass values are model dependent and should be taken with some caveats. Since the first spectroscopic

Send offprint requests to: D. Barrado y Navascués, e-mail: barrado@pollux.ft.uam.es

* These observations were collected at the VLT of the European Southern Observatories. confirmation of the nature of some IPMOs in $\sigma$ Orionis and the Trapezium (Zapatero Osorio et al. 2000; Lucas et al. 2001), they have become very interesting for two different reasons: (i) they extend the sequence of low mass objects beyond the deuterium burning threshold (Saumon et al. 1996; Chabrier et al. 2000); and (ii) their very existence poses a challenge to our understanding of how they have been created during the collapse and fragmentation of molecular clouds, since so far no model has been able to predict the formation of objects in isolation in this low mass range (e.g. Bodenheimer 1998). Other alternatives have been recently suggested, such as the formation in multiple systems as stellar embryos and the ejection from the system before they accrete enough material to become stars or brown dwarfs (Boss 2001; Reipurth 2001; Bate 2001).

Here, we present low resolution optical spectroscopy of isolated planetary mass candidates in the $\sigma$ Orionis cluster. In our analysis, we assume a likely cluster age of $5 \mathrm{Myr}$, which relies on several observational facts combined with theory: (i) the 5 Myr-isochrone provides the best fit to the location of stellar and substellar $\sigma$ Orionis members in color-magnitude diagrams (Béjar et al. 2001), (ii) there is no evidence of lithium depletion in low mass stellar members indicating that the cluster is younger than 

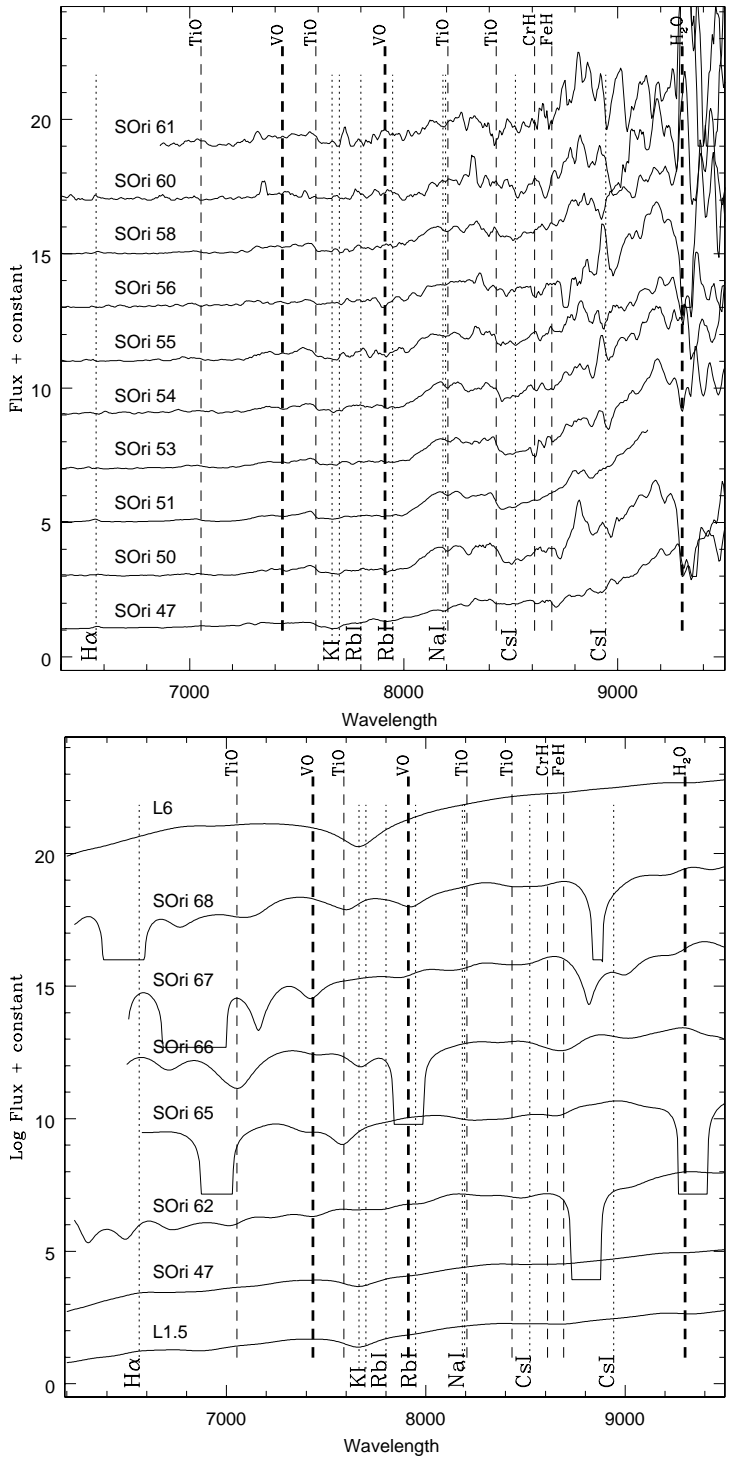

Fig. 1. VLT/FORS spectra of isolated planetary mass candidate members of the $\sigma$ Orionis cluster. Top panel: bright objects, data smoothed using a boxcar of 5 pixels. Lower panel: faint objects, data smoothed using a boxcar of 5 pixels and a Gaussian function with a 12 sigma kernel. Note the logarithmic scale in the $y$-axis. For comparison, we include two L1.5- and L6-type field objects and S Ori 47.

$8 \mathrm{Myr}$ in order to account for total lithium preservation (Zapatero Osorio et al. 2001), (iii) high mass star models including mass loss calculations (Meynet et al. 2001) predict that the central multiple star ( $\sigma$ Orionis itself, 09.5 V, still burning hydrogen on the main sequence phase) has to be younger that about $7 \mathrm{Myr}$. Therefore, we believe the age of $5 \mathrm{Myr}$ is very realistic, being $7 \mathrm{Myr}$ an upper limit for the age of the association.

\section{Observations}

Our spectroscopic data were collected with the Very Large Telescope Unit \#1 at the Paranal Observatory of the European Southern Observatory during Dec. 23-27, 2000.
Table 1. $\sigma$ Orionis member candidates with derived masses below the deuterium burning mass limit (for an age of $5 \mathrm{Myr}$ ).

\begin{tabular}{lccccl}
\hline Name & $I$ & $I-J$ & $\begin{array}{c}W_{\lambda}(\mathrm{H} \alpha) \\
(\AA)\end{array}$ & Sp.Type & $\begin{array}{l}\text { Sp.Type } \\
\text { previous }\end{array}$ \\
\hline SOri47 & 20.53 & 3.15 & 25 & $\mathrm{~L} 1.0 \pm 1.0$ & $\mathrm{~L} 1.5$ \\
SOri50 & 20.66 & 3.12 & $<10$ & $\mathrm{M} 9.0 \pm 0.5$ & \\
SOri51 & 20.71 & 3.50 & 25 & $\mathrm{M} 9.0 \pm 0.5$ & \\
SOri53 & 21.17 & 3.28 & $<10$ & $\mathrm{M} 9.0 \pm 0.5$ & \\
SOri54 & 21.29 & 3.30 & 15 & $\mathrm{M} 9.5 \pm 0.5$ & \\
SOri55 & 21.32 & 3.10 & 5 & $\mathrm{M} 9.0 \pm 1.0$ & \\
SOri56 & 21.74 & 3.30 & $<10$ & $\mathrm{~L} 1.0 \pm 1.5$ & $\mathrm{~L} 0.5$ \\
SOri58 & 21.90 & 3.30 & 25 & $\mathrm{~L} 0.0 \pm 1.0$ & \\
SOri60 & 22.75 & 3.58 & $\sim 25$ & $\mathrm{~L} 2.0 \pm 0.5$ & \\
SOri61 & 22.78 & 3.16 & - & $\mathrm{L} 0.0 \pm 1.5$ & \\
SOri62 & 23.03 & 3.59 & $\sim 50$ & $\mathrm{~L} 2.0 \pm 1.5$ & $\mathrm{~L} 4.0$ \\
SOri65 & 23.23 & 3.33 & $<20$ & $\mathrm{~L} 3.5 \pm 2.0$ & \\
SOri66 & 23.23 & 3.40 & $\sim 100$ & $\mathrm{~L} 3.5 \pm 2.0$ & \\
SOri67 & 23.40 & 3.49 & $\sim 50$ & $\mathrm{~L} 5.0 \pm 2.0$ & \\
SOri68 & 23.77 & 3.59 & $<20$ & $\mathrm{~L} 5.0 \pm 2.0$ & \\
\hline & & & & &
\end{tabular}

We used the FORS1 spectrograph and the multi slit capability. FORS1 has a $0.2^{\prime \prime} /$ pixel scale in the standard resolution, yielding a field of view of $6.8^{\prime} \times 6.8^{\prime}$. For our spectroscopic data, we used the 150I grism and the order-blocking filter OG590. With a slit width of $1.4^{\prime \prime}$, our resolution is $R \sim 250$, as measured in the comparison arcs. Our sample of $\sigma$ Orionis IPMOs was selected from Zapatero Osorio et al. (2000). In total, we observed 14 out of the 18 original objects, together with S Ori 47, discovered previously (Zapatero Osorio et al. 1999). Table 1 lists magnitudes and colors. Exposure times are in the range 2400-16800 s.

The data were reduced using standard procedures within the IRAF environment. Individual exposures were added together. Then, the spectra were extracted using the "apall" package within IRAF, fitting the sky to remove the emission lines and the background. The wavelength calibration was performed using $\mathrm{HeArHgCd}$ comparison arcs taken with the same configuration. Then data were flux calibrated using spectrophotometric standards. Finally, we improved the signal to noise ratio $(S / N)$ by smoothing the spectra with a boxcar of 5 pixels $(R \sim 210)$. The spectra corresponding to faintest objects were also convolved with a Gaussian function of 12 sigma kernels $(R \sim 60)$. Figure 1 displays the spectra of our $\sigma$ Orionis targets. The upper panel shows the brightest targets $\left(I_{\mathrm{C}}=20.5-22.8 \mathrm{mag}\right)$, with a good signal to noise ratio $(S / N=100-40)$. while the lower panel displays fainter objects, down to $I=23.77 \mathrm{mag}$, with a much poorer quality. Note that when, due to the generally low $S / N$ at the bottom of the molecular bands, the subtraction of the sky spectrum was not good enough, we have modified artificially the bad spectral range. These areas are replaced with horizontal segments in the modified spectra. In addition to the $\sigma$ Orionis targets, we observed several nearby field objects of $\mathrm{M}$ and $\mathrm{L}$ spectral type, for comparison purposes (to derive spectral types). 


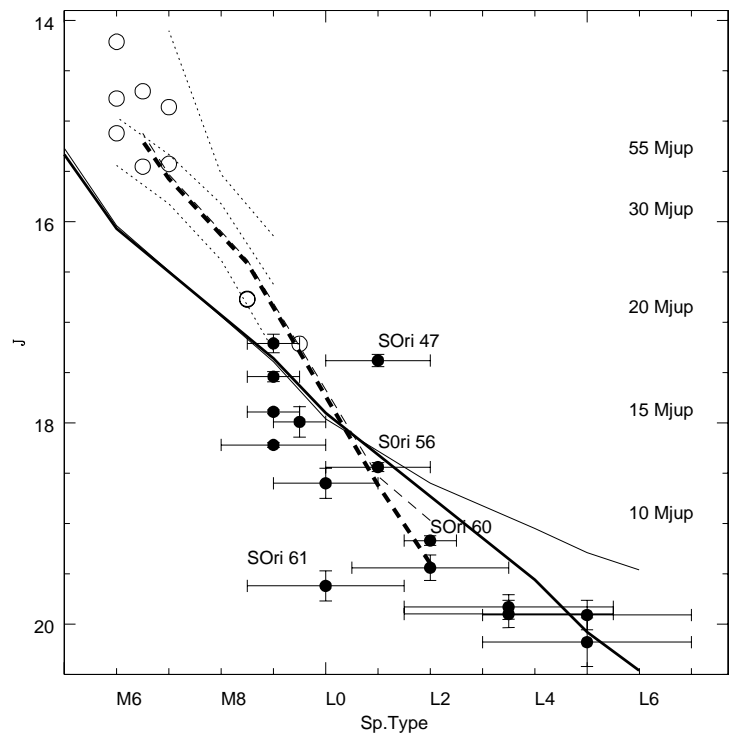

Fig. 2. Spectral type against $J$ magnitude. Solid circles represent data from this study, whereas open circles correspond to data from Béjar et al. (2001). The location of the brown dwarfplanetary mass domain borderline in $\sigma$ Orionis is at around $J=17.9 \mathrm{mag}$ at the age of $5 \mathrm{Myr}$. The lines represent several 5 Myr isochrones from Baraffe et al. (1998) - thin lines - and Chabrier et al. (2000) - thick lines, which were obtained for different temperature scales (low gravity by Béjar et al. $2001-$ dashed lines; high gravity by Basri et al. 2000 - solid lines, and different gravities by Luhman 1999 - dotted lines). See text.

\section{Spectral types}

Figure 1 also depicts several relevant spectral features observable in the optical, like lines from alkali elements (K I $\lambda 7665$ and $\lambda 7699 \AA$, Na I $\lambda 8183$ and $\lambda 8195 \AA$, Cs I $\lambda 8521$ and $\lambda 9843 \AA$, and Rb I $\lambda 7800$ and $\lambda 7948 \AA$, dotted vertical lines), and molecular absorptions of $\mathrm{TiO}, \mathrm{FeH}, \mathrm{CrH}$ (vertical thin dashed lines), and of $\mathrm{VO}$ and $\mathrm{H}_{2} \mathrm{O}$ (vertical thick dashed lines). In particular, the differences can be appreciated, both in the slope of the pseudo-continuum as well as the change in the strength and width of the $\mathrm{K}$ I resonance doublet, $\mathrm{VO}$ and $\mathrm{TiO}$ bands, etc. Detailed studies of characteristics of late $\mathrm{M}$ and field $\mathrm{L}$ very low mass stars and brown dwarfs can be found in Kirkpatrick et al. (1999) and Martín et al. (1999). Figure 1 indicates that, with this resolution, it is possible to distinguish key spectral features and attempt a spectral classification of the $\sigma$ Orionis candidate members.

Spectral types have been assigned following the scheme proposed in Martín et al. (1999). We have measured flux ratios between several bands (pseudo-continuum, VO, $\mathrm{TiO}$, etc.) and compared them with M- and L-type, nearby field objects. For the faintest object in the sample, having a low $S / N$, we derived a spectral type based on the slope of the continuum. Our final spectral types and uncertainties are listed in Table 1. Note that the indices we have used, in particular PC3 (Martín et al. 1996), are based on field, older objects, which have higher surface gravity than members of $\sigma$ Orionis. Evolutionary models predict gravities about $\log g=3.5-4.0$ for very low mass objects at young ages around $5 \mathrm{Myr}$. L-type sources (very cool temperatures) display optical energy distributions characterized by strong atomic lines of Na I and K I (Allard et al. 2001; Pavlenko et al. 2000), which markedly dominate the shape of the far-red wavelengths. These lines are very sensitive to gravity (Allard et al. 2001): low gravity Ltype spectra display significantly less intense alkaline lines. Because the blue side of the PC3 index is located very close to the K I resonance doublet, it provides earlier L spectral types for low gravity objects than for high gravity ones. The M-classes are not so much affected (Béjar 2001) by the gravity effect. On the contrary, the near-infrared spectra of these types are governed by water vapor absorptions, which are steeper for low gravities (Allard et al. 2001). Our latest optical spectral types appear shifted by 1-2 subclasses towards warmer L-types compared to the nearinfrared classification given in Martín et al. (2001) for the objects in common.

The objects in our target list define a neat spectral sequence: the fainter the magnitude and redder the color, the cooler the spectral class. We show the relation between spectral type and $J$ magnitude in Fig. 2. Open circles correspond to $\sigma$ Orionis brown dwarfs from Béjar et al. (2001), whereas solid circles stand for the data studied here. Estimated masses for each magnitude appear on the right-hand side of the diagram on the basis of the $5 \mathrm{Myr}$ dusty isochrone by Chabrier et al. (2000). Albeit this is the likely age of the cluster (see Sect. 1), we note that the location of the borderline between brown dwarfs and IPMOs $(J \sim 17.9 \mathrm{mag}$ at $5 \mathrm{Myr})$ does not change by a large amount for other possible ages such as $3 \mathrm{Myr}(J \sim$ $17.5 \mathrm{mag}), 7 \mathrm{Myr}(J \sim 18.2 \mathrm{mag})$, and the oldest value of $10 \operatorname{Myr}(J \sim 18.6 \mathrm{mag})$.

For comparison purposes, Fig. 2 also includes several $5 \mathrm{Myr}$ isochrones using the grainless models of Baraffe et al. (1998) and dusty models of Chabrier et al. (2000). These models provide magnitudes in the filters of interest and are represented as thin and thick lines, respectively. Essentially, there is no difference in this diagram between dusty and grainless models for objects warmer than about L1. We have adopted several calibrations in order to derive spectral types from effective temperatures:

(i) Leggett et al. (2000) for early- to mid-M types and Basri et al. (2000) for later classes, which appear as solid lines;

(ii) Béjar (2001), shown as dashed lines (see below);

(iii) Luhman (1999), represented as dotted lines. In this last case, calibrations for giant, intermediate and dwarf objects have been included (from top to bottom).

This last set of isochones shows the effect of gravity: the lower the gravity, the more luminous the object is in the $J$ band. Leggett et al. (2000) and Basri et al. (2001) obtained their temperature scale by studying objects in the field and assuming gravities in the range $\log g=5.0-5.5$, whereas Béjar's scale was derived for the lower gravity 
of $\log g=3.5$, which is more appropiate for members of the young $\sigma$ Orionis cluster. This late author compared observed low resolution optical spectra of cluster M-type brown dwarfs and S Ori 47 to spectral synthesis computed for the dusty model atmospheres of Allard et al. (2001) following the prescriptions given in Pavlenko et al. (2000). The agreement between observations and isochrones of Fig. 2 is better for the Béjar's (2001) temperature scale. We note that the temperature calibration of Luhman (1999), which is determined for gravities intermediate between those of dwarfs and giants, also provides a reasonable fit to our observations - objects with spectral types earlier than M9 - and is indeed similar to that of Béjar (2001) in this spectral range. Finally, dusty models seem to fit better the observations for objects having spectral types later than L1. In any case, the current uncertainties involved in the conversion between effective temperatures and spectral types are still rather high and we believe more data are needed to better constrain temperature scales for objects of different gravities.

As seen from Fig. 2, there is a clear, monotonic spectral sequence from mid-M ( $\sigma$ Orionis massive brown dwarfs) to mid-L ( $\sigma$ Orionis IPMOs). However, there are few exceptions. S Ori 61 deviates from the cluster sequence since it shows a magnitude fainter than expected for its spectral class. Thus, we cannot confirm its membership in the cluster. On the other hand, S Ori 47 clearly stands out among other $\sigma$ Orionis members. The quality of our data, and previously published data by Zapatero Osorio et al. (1999, $2000)$, confirms that this object, with a magnitude around the deuterium burning mass limit at the age of the cluster, has a spectral type of L1.0-1.5. Actually, S Ori 47 could be a nearly equal mass binary comprised of two IPMOs. If this is later confirmed with follow-up observations, this object would become the first binary IPMO with components of 9-13 $M_{\text {Jupiter }}$ each for the age range of $1-7 \mathrm{Myr}$. S Ori 56 and S Ori 60 also appear too bright for their spectral types in Fig. 2. Moreover, S Ori 60 shows overluminous and with very red colors in infrared color-magnitude diagrams. Our estimated binarity fraction (3 out of 18) in the very low-mass regime of the $\sigma$ Orionis cluster agrees with recent observations of binaries among field L-dwarfs (about 20\%, see Reid et al. 2000). A search for visual companions around $\sigma$ Orionis IPMOs using $K$-band imaging can be found in Martín et al. (2001).

\section{4. $\mathrm{H} \alpha$, membership and the initial mass function}

The equivalent width of the $\mathrm{H} \alpha$ line at $\lambda 6563 \AA$ is considered as an age indicator in M-dwarfs, and it is usually associated to stellar activity. In general, the stronger the emission line for a given spectral type, the younger the object. We have identified this feature in emission for the first time in such low mass objects, and measured its equivalent width $(E W)$. The results are listed in Table 1. For those objects we do not see $\mathrm{H} \alpha$ in emission we can impose an upper limit to the $E W$ at $10 \AA$ and $20 \AA$, depending on their brightness. Nine objects out of 14 (the spectrum
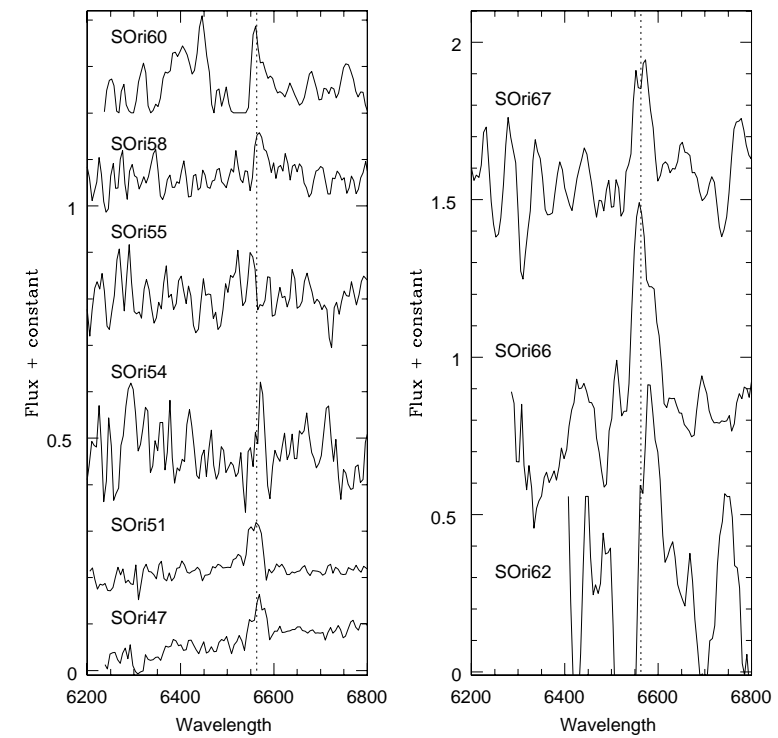

Fig. 3. Detail around the $\mathrm{H} \alpha$ line. No smoothing or convolution with Gaussian functions have been performed.

of one of them does not reach this wavelength) have a significant emission in $\mathrm{H} \alpha$, a strong evidence that they are young and probable members of the stellar association. A close-up of the area around $\mathrm{H} \alpha$ is depicted in Fig. 3. We note that very few $\mathrm{H} \alpha$ emissions have been detected in similar spectral type field objects (Kirkpatrick et al. 1999; Gizis et al. 2000), and the $E W$ s of the lines are typically below $10 \AA$. The origin of this feature is not clear for IPMOs. Actually, the emission could be due to the presence of mass accretion from a gas-dust disk (Muzerolle et al. 2000). If this is true this fact might be indicating that these objects have formed in isolation by direct collapse and cloud fragmentation, and they are not runaways from embryonic multiple systems. A diagram illustrating the $\mathrm{H} \alpha$ behaviour as a function of $(I-J)$ color is presented in Fig. 4. It seems that $\mathrm{H} \alpha$ emission is larger for cooler objects. One of our targets, SOri 55, may have experienced a flare-like episode displaying a considerable variability in the $\mathrm{H} \alpha E W$ (Zapatero Osorio et al. 2001). Moreover, S Ori 47 also has a variable $\mathrm{H} \alpha$ emission, since we measure a $E W$ of $25 \AA$ on the VLT spectrum while Zapatero Osorio et al. (1999) found an upper limit of $6 \AA$.

All the available information, both spectroscopic and photometric, indicates that most of the objects in our sample are bona-fide members, with masses in the planetary domain and slightly above it. Recently, Béjar et al. (2001) have derived a initial mass function (IMF) for the substellar domain of the $\sigma$ Orionis cluster, finding $\alpha=0.8 \pm 0.4$ (where $\mathrm{d} N / \mathrm{d} M=k M^{-\alpha}$ ). Our data confirm that the smallest mass bin of that IMF, corresponding to planetary mass members, is essentially unaffected by photometric contamination, i.e., interlopers. Therefore, the cluster IMF keeps rising below the deuterium burning limit, implying that the cluster contains a large number of brown dwarfs and IPMOs. 


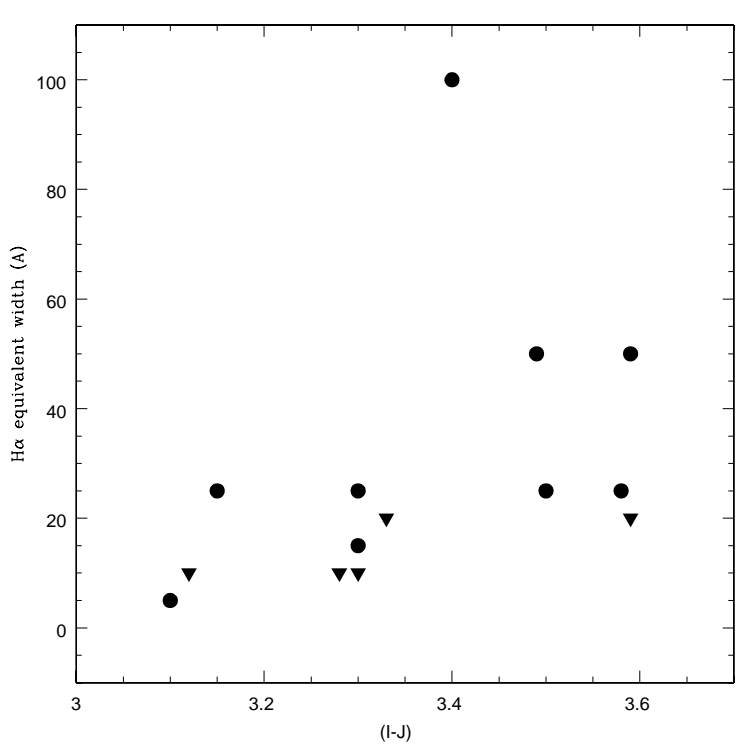

Fig. 4. $\mathrm{H} \alpha$ equivalent width against the color $(I-J)$. Detections and upper limits appear as circles and triangles, respectively.

\section{Conclusions}

Using VLT/FORS1 low resolution spectrograph, we have derived spectral types for 15 isolated planetary mass object (IPMO) candidates in the $\sigma$ Orionis cluster discovered by Zapatero Osorio et al. (2000). All of these objects but one (S Ori 61) appear to be bona fide members of the cluster, since they follow a well defined sequence in the magnitude versus spectral type and color diagrams. A significant fraction of the IPMOs presents $\mathrm{H} \alpha$ in emission, and at least two of them show variability in this activity indicator (S Ori 47 and S Ori 55). Since their membership in the cluster is confirmed with our optical spectroscopic data, the masses of these planetary objects must be in the range $18-8 M_{\text {Jupiter }}$ adopting a cluster age of $5 \mathrm{Myr}$. The combined information provided by the color-magnitude and the spectral type-magnitude diagrams indicates that S Ori 47 is a likely photometric binary. Other two objects (SOri 56 and S Ori60) might also be photometric binaries. The spectra presented here prove that membership is essentially correct in Zapatero Osorio et al. (2000). Therefore, the substellar mass function derived by Béjar et al. (2001), is not biased by spurious members in the planetary mass domain.

Acknowledgements. We thanks the ESO staff at Paranal Observatory. The referee, G. Chabrier, has helped to improve the original version with his comments and suggestions. Partial financial support was provided by the Spanish DGES project PB98-0531-C02-02 and CICYT grant ESP98-1339-CO2.

\section{References}

Allard, F., Hauschildt, P. H., Alexander, D. R., Tamanai, A., \& Schweitzer, A. 2001, ApJ, 556, 357

Baraffe, I., Chabrier, G., Allard, F., \& Hauschildt, P. H. 1998, A\&A, 337, 403

Basri, G., Mohanty, S., Allard, F., et al. 2000, ApJ, 538, 363

Bate, M. 2001, in The origins of Stars and Planets: the VLT view, ed. J. Alves, \& M. McCaughrean (Springer-Verlag), series ESO Astrophys. Symp., in press

Béjar, V. J. S. 2001, Ph.D. dissertation, Universidad de La Laguna, Spain

Béjar, V. J. S., Martín, E. L., Zapatero Osorio, M. R., et al. 2001, ApJ, 556, 830

Bodenheimer, P. 1998, in ASP Conf. Ser. 134, Brown Dwarfs and Extrasolar Planets, ed. R. Rebolo, E. L. Martín, \& M. R. Zapatero Osorio (San Francisco: ASP), 115

Boss, A. P. 2001, ApJ, 551, L167

Chabrier, G., Baraffe, I., Allard, F., \& Hauschildt, P. 2000, ApJ, 542, L119

Gizis, J. E., Monet, D. G., Reid, I. N., et al. 2000, AJ, 120, 1085

Kirkpatrick, D., Reid, I. N., Liebert, J., et al. 1999, ApJ, 519, 802

Leggett, S. K., Allard, F., Dahn, C., et al. 2000, ApJ, 535, 965

Lucas, P. W., \& Roche, P. F. 2000, MNRAS, 314, 858

Lucas, P. W., Roche, P. F., Allard, F., \& Hauschildt, P. H. 2000, MNRAS, 326, 695

Luhman, K. L. 1999, ApJ, 525, 466

Martín, E. L., Rebolo, R., \& Zapatero Osorio, M. R. 1996, ApJ, 469, 706

Martín, E. L., Delfosse, X., Basri, G., et al. 1999, AJ, 118, 2466

Martín, E. L., Zapatero Osorio, M. R., Barrado y Navascués, D., Béjar, V. J. S., \& Rebolo, R. 2001, ApJL, accepted

Meynet, G., Maeder, A., Schaller, G., Schaerer, D., \& Charbonnel, C., 1994, A\&AS, 103, 97

Muzerolle, J., Briceño, C., Calvet, N., et al. 2001, ApJ, 545, L141

Najita, J., Tiede, G. P., \& Carr, J. S. 2000, ApJ, 541, 977

Pavlenko, Ya., Zapatero Osorio, M. R., \& Rebolo, R. 2000, A\&A, 355, 245

Reid, I., Gizis, J. E., Kirkpatrick, J. D., \& Koerner, D. W. 2001, AJ, 121, 489

Reipurth, B. 2001, in The origins of Stars and Planets: the VLT view, ed. J. Alves, \& M. McCaughrean (Springer-Verlag), series ESO Astrophys. Symp., in press

Saumon, D., Hubbard, W. B., Burrows, A., et al. 1996, ApJ, 460,993

Zapatero Osorio, M. R., Béjar, V. J. S., Rebolo, R., Martín, E. L., \& Basri, G. 1999, ApJ, 524, L115

Zapatero Osorio, M. R., Béjar, V. J. S., Martín, E. L., et al. 2000, Science, 290, 103

Zapatero Osorio, M. R., et al. 2001, in preparation 